\title{
Increased Biglycan in Aortic Valve Stenosis Leads to the Overexpression of Phospholipid Transfer Protein via Toll-Like Receptor 2
}

\author{
Habib Derbali, ${ }^{*}$ Yohan Bossé, ${ }^{\dagger}$ Nancy Côté, ${ }^{*}$ \\ Philippe Pibarot, ${ }^{\dagger}$ Audrey Audet, ${ }^{*}$ Andree Pépin, ${ }^{*}$ \\ Benoit Arsenault, ${ }^{\ddagger}$ Christian Couture, ${ }^{\S}$ \\ Jean-Pierre Després, ${ }^{\ddagger}$ and Patrick Mathieu* \\ From the Laboratoire d'Études Moléculaires des Valvulopathies \\ (LEMV), Groupe de Recherche en Valvulopathies (GRV), Institut \\ Universitaire de Cardiologie et de Pneumologie de Québec/ \\ Research Center, Department of Surgery,* the Department of \\ Medicine, the Division of Kinesiology, the Department of Social \\ and Preventive Medicine, ${ }^{\ddagger}$ and the Department of Pathology, \\ Laval University, Québec, Canada
}

Aortic stenosis (AS) is the most common valvular heart disease, and it is suspected that atherosclerotic mechanisms are involved in the development of this disorder. Therefore, the retention of lipids within the aortic valve may play a role in the pathobiology of AS. In this study, a gene expression microarray experiment was conducted on human aortic valves with and without AS. The expression levels of transcripts encoding proteoglycans and enzymes involved in lipid retention were compared between the two groups. The microarray results were subsequently replicated in a cohort of $87 \mathrm{AS}$ valves and 36 control valves. In addition, the interaction between proteoglycan and lipid-modifying enzyme was documented in isolated valve interstitial cells (VICs). The microarray results indicated that only biglycan (BGN) and phospholipid transfer protein (PLTP) were overexpressed in the AS valves. These results were then confirmed by quantitative PCR. The immunohistochemical analysis revealed a colocalization of BGN, PLTP, and Toll-like receptor-2 (TLR 2) in AS valves. In vitro, we showed that BGN induces the production of PLTP in VICs via the stimulation of TLR 2 . Thus, increased accumulation of BGN in AS valves contributes to the production of PLTP via TLR 2. These results suggest that intricate links between valve matrix proteins, inflammation, and lipid retention are involved in the pathobiology of AS. (Am J Pathol 2010, 176:2638-2645; DOI: 10.2353/ajpath.2010.090541)
Calcific aortic stenosis (AS), a common heart valve disorder, shares several similarities with vascular atherosclerorosis. Indeed, previous studies have underlined that age, male gender, hypertension, diabetes, and metabolic syndrome were associated with AS. ${ }^{1}$ Furthermore, histological studies have pinpointed that, besides tissue mineralization, accumulation of lipids along with inflammation are key features of $\mathrm{AS}^{2}{ }^{2}$ More recently, Toll-like receptors (TLRs) have been detected in AS valves as well as in isolated valve interstitial cells (VICs), where they are believed to play an important role in the pathophysiology of this disease. ${ }^{3}$ In this regard, activation of TLR 2 and 4 has been shown to be involved in the production of cytokines and bone-related proteins. ${ }^{4}$

Biglycan (BGN) is a proteoglycan that by its affinity to lipoproteins promotes lipid retention within the atherosclerotic plaque. ${ }^{5}$ In turn, lipid retention allows biochemical modifications of lipoproteins by enzymes, such as lipoprotein lipase (LPL), hepatic lipase (LIPC), and phospholipid transfer protein (PLTP). ${ }^{6}$ The presence of PLTP has been demonstrated in the atherosclerotic plaque, where it modifies lipoproteins such as Apo Al, which, in turn, has a greater affinity for proteoglycans. ${ }^{7}$ Recently, a study has underlined that BGN binds to murine TLR 2 and 4, whereby it induces the production of inflammatory

Supported by the Canadian Institute of Health Research (CHR), Ottawa, Canada (grant number MOP 79342), the Quebec Heart Institute Foundation, and the Réseau d'Échanges et de Tissus Biologiques, Fonds de Recherche en Santé du Québec, Montreal, Canada. P.P. holds the Canada Research Chair in Valvular Heart Diseases, Canadian Institutes of Health Research, Ottawa, Ontario, Canada. J.-P.D. is the Scientific Director of the International Chair on Cardiometabolic Risk at University Laval, which is supported by an unrestricted grant from Sanofi-Aventis. P.M. is a research scholar from the Fonds de Recherches en Santé du Québec, Montreal, Canada

Accepted for publication February 16, 2010

Supplemental material for this article can be found on http://ajp. amjpathol.org

Address reprint requests to Dr. Patrick Mathieu, Institut Universitaire de Cardiologie et de Pneumologie de Québec, 2725 Chemin Ste-Foy Québec, Quebec, Canada, G1V-4G5. E-mail: patrick.mathieu@chg.ulaval.ca. 
cytokines through the nuclear factor kappa B (NF- $\kappa \mathrm{B})$ pathway. ${ }^{8}$

A majority of explanted human AS valves are infiltrated with oxidized-LDL. In addition, a recent study has demonstrated that greater accumulation of oxidized lipids within the aortic valve is associated with more pronounced valvular inflammation and faster progression rate of the stenosis. ${ }^{2}$ Of interest, in the latter study the proportion of small dense LDL in the plasma was the only blood lipid variable associated with the valvular accumulation of lipids. It is thus likely that mechanisms pertaining to lipid retention and modifications within AS valves play a key role in pathogenesis of AS. The presence of proteoglycans, LPL, LIPC, and PLTP within the valve as well as their relationships with blood lipid variables has never been explored in patients with AS. Hence, the objective of this study was to determine whether proteoglycans were increased in AS valves and would be related to plasma lipid variables. In addition, we tested whether BGN might mediate through TLR the production of lipidmodifying enzyme by VICs.

\section{Materials and Methods}

\section{Patients and Tissue Collection}

We examined 87 aortic valves explanted from patients who underwent aortic valve replacement for AS. Thirty six aortic valves with normal morphology and function were obtained from patients undergoing a heart transplantation procedure and were used as controls. The protocol was approved by local ethical committee, and informed consent has been obtained from the subjects. All patients had moderate to severe AS. Patients with more than mild aortic regurgitation or with a history of rheumatic disease, endocarditis, or an inflammatory disorder were excluded. Samples were taken at the time of surgery. One cusp was snap frozen in liquid nitrogen for ulterior RNA isolation by quantitative PCR analyses. Two cusps were decalcified in Cal-Ex (Fisher, Ottawa, ON, Canada) for 24 hours and then were embedded in optimum cutting temperature (OCT) compound (Somagen diagnostics, Edmonton, Alberta, Canada) and frozen in liquid nitrogen for immunohistological analysis.

\section{Microarray}

A total of five AS valves and five normal aortic valves were selected for microarray application. To reduce heterogeneity, only valves taken from male subjects were selected. The two valve groups were also matched for patient's age and body mass index. Expression studies were performed using the human U133 plus 2.0 Affymetrix GeneChip microarrays (Affymetrix, Santa Clara, CA). Arrays were processed using a standard Affymetrix double amplification protocol using $80 \mathrm{ng}$ of RNA. Expression values were extracted using the Robust Multichip Average (RMA) method. Quality control assessment was performed with the FlexArray software version RC3 and the affy package that is part of the Bioconductor project (http://www.bioconductor.org/). Two microarrays interrogating control valves failed quality control and were discarded from the analysis. Significant analysis of microarrays (SAM) method was used to claim significant regulation. The minimum fold change and the delta values was set to 2 and 0.76 , respectively, to reach a false discovery rate below $5 \%(4.85 \%)$. The microarray dataset can be found in the National Center for Biotechnology Information's Gene Expression Omnibus (GEO) repository (http://www.ncbi.nlm.nih.gov/geo/) and are accessible through GEO Series accession number GSE12644.

\section{RNA Extraction and Real-Time PCR}

In 87 AS valves and 36 controls, RNA was extracted. RNA was also extracted from isolated VICs. Total RNA was isolated with RNeasy micro kit from Qiagen (Qiagen, Mississauga, ON, Canada). The RNA extraction protocol was performed according to manufacturer's instructions using $100 \mathrm{mg}$ of tissue. The quality of total RNA was monitored by capillary electrophoresis (Experion, Biorad, Mississauga, ON, Canada). Four $\mu \mathrm{g}$ of RNA was reverse transcribed using the Quantitec Reverse Transcription Kit from Qiagen. Quantitative PCR was performed with Quantitec SYBR Green PCR kit from Qiagen in the RotorGene 6000 system (Corbett Robotics Inc, San Francisco, CA). BGN, PLTP, and NF- $\kappa B$ (Qiagen, Mississauga, ON, Canada) were quantified with real-time quantitative PCR at the following conditions: an initial 15 minutes run at $95^{\circ} \mathrm{C}$ before starting, then $94^{\circ} \mathrm{C}$ for 10 seconds, $55^{\circ} \mathrm{C}$ for 30 seconds, and $72^{\circ} \mathrm{C}$ for 30 seconds for a total of 40 cycles. The expression of a reference gene, HPRT (coding: 5'-TGGCGTCGTGATTAGTGATG-3', noncoding: 5'AATCCAGCAGGTCAGCAAAG-3'), was chosen as a normalizer to control for any difference in the amount of cDNA starting material. The real-time PCR for HPRT was done at the following conditions: an initial 15 minutes run at $95^{\circ} \mathrm{C}$ before starting, then $94^{\circ} \mathrm{C}$ for 10 seconds, $58^{\circ} \mathrm{C}$ for 30 seconds, and $72^{\circ} \mathrm{C}$ for 30 seconds for a total of 40 cycles. Both primers were home designed and synthesized by Invitrogen (Invitrogen, Toronto, ON, Canada).

\section{Immunohistology and Quantitative Morphometric Analyses}

Immunohistology was performed in cryostat sections using the following mouse Mab: BGN (R\&D systems inc, Minneapolis, MN), PLTP (Abnova, Taipei City, Taiwan), TLR 2 (BioLegend, San Diego, CA), TLR 4 (Abcam, Cambridge, MA,USA), Apo Al (AbD Serotec, Oxford, UK), $\alpha$-actin (Sigma-Aldrich, Oakville, ON, Canada), and CD90 (VWR International, Mississauga, ON, Canada). The presence of oxidized-LDL (ox-LDL) was also detected using immunohistologic analysis with the following antibody: a polyclonal (rabbit) antibody anti-OX-LDL (Calbiochem, Hornby, ON, Canada). For the single staining, slides were incubated with EnVision Dual Link System-HRP followed by AEC substrate (Dako, Carpinteria, $\mathrm{CA})$. For the simultaneous detection of two different antigens within the same specimen, slides were treated with 
EnVision G2 Doublestain System. Then, they were processed with DAB (brown coloration) and the Permanent Red chromogenic substrate (Dako, Carpinteria, CA). Tissue sections were counterstained with hematoxylin. Mouse or rabbit serum were used as a negative control.

The presence of BGN and PLTP was detected by immunostaining and quantitative analyses in 36 control valves and 41 AS valves. The area of each valve was determined using the Image Pro Plus Version 6.1 image analysis software. The surface of BGN was measured on the entire valve section. Then, the percentage of BGN immunostaining on the total area of the valve was calculated. For the quantification of PLTP, each cells positively marked on the tissue were counted at $\times 400$ and reported on the area of the valve.

\section{Valve Interstitial Cells Isolation and in Vitro Analyses}

Human VICs were isolated by collagenase digestion from explanted hearts during transplant procedures. Cells were cultured in DMEM containing 10\% FBS, $2 \mathrm{mmol} / \mathrm{L}$ L-glutamine, and $1 \mathrm{mmol} / \mathrm{L}$ sodium pyruvate (Invitrogen, Toronto, ON, Canada). Cells were seeded in 12-well plates $\left(2 \times 10^{4}\right.$ cells/well). In some experiments VICs were stimulated with BGN $(50,100 \mathrm{ng} / \mathrm{ml})$, bacterial lipopolysaccharide (LPS; Sigma-Aldrich, Oakville, ON, Canada; $200 \mathrm{ng} / \mathrm{ml}$ ), or peptidoglycan from Staphylococcus aureus (PGN; Sigma-Aldrich, Oakville, ON, Canada; 10 $\mu \mathrm{g} / \mathrm{ml})$. Also, in a series of experiments VICs were stimulated with BGN (100 ng/ml) with or without TLR 2, TLR 4 antibodies (BioLegend, San Diego, CA; $10 \mu \mathrm{g} / \mathrm{ml}$ ), or a control irrelevant human antibody $\lg _{1} \mathrm{Fc}_{\mathrm{c}}$ (R\&D systems, Minneapolis, MN). Cells were stimulated for 48 hours and protein or RNA isolated for analyses.

In a another series of experiments, human VICs were seeded in 12-well plates $\left(2 \times 10^{4}\right.$ cells per well $)$ and treated with HiPerFect from Qiagen (Mississauga, ON, Canada). Cells were then transfected with TLR 2, TLR 4, or control siRNA (Qiagen, Mississauga, ON, Canada). After 48 hours, cells were stimulated with BGN (100 ng/ ml; Sigma-Aldrich, Oakville, ON, Canada) for 48 hours and RNA isolated.

To measure NF- $\kappa \mathrm{B}$ activity in VICs stimulated with BGN, we have measured levels of phospho-NF- $\kappa \mathrm{B}$ with and without siRNA for TLR 2. After 48 hours of treatment with or without BGN $(100 \mathrm{ng} / \mathrm{ml})$ cells were harvested in $400 \mu \mathrm{l}$ of ice-cold lysis buffer containing $1 \mathrm{mmol} / \mathrm{L}$ phenylmethysulfonylfluoride (PMSF). Cells were then sonicated and centrifuged at $4^{\circ} \mathrm{C}(14,000 \mathrm{~g}, 10$ minutes). The supernatant was used for detection of NF- $\kappa \mathrm{B}$ activity. The total and phospho NF- $\kappa$ B levels were measured by ELISA with the PathScan Total NF- $\kappa \mathrm{B} 65$ and the PhosphoNF- $\kappa$ B 65 sandwich ELISA kit from Cell Signaling Technology (New England Biolabs, Pickering, ON, Canada).

\section{Western Blot}

VICs were harvested in $200 \mathrm{mmol} / \mathrm{L}$ Tris- $\mathrm{HCl} \mathrm{Ph} 6.8,2 \%$ SDS + protease inhibitor (Roche diagnostic, Laval, QC,
Canada). The protein concentration of supernatant was determined with DC protein assay from Bio-Rad. Proteins were loaded onto 6\% SDS-polyacrylamide gels followed by electrophoresis and blotting onto nitrocellulose membranes. Membranes were blocked with TBS-tween containing $5 \%$ nonfat dry milk, incubated with mouse monoclonal antibody directed against PLTP (Sigma-Aldrich, Oakville, ON, Canada) and $\beta$-Actin (Sigma-Aldrich, Oakville, ON, Canada), followed by an HRP-labeled antimouse IgG antibody (Amersham, Piscataway, NJ). Detection was done with Supersignal West Pico chemiluminescent substrate (Pierce Lab, Rockford, IL).

\section{Lipid Profile}

Overnight fasting plasma was collected and immediately processed by the laboratory for the measurement of glucose, total cholesterol, low-density lipoprotein cholesterol (LDL-C), high-density lipoprotein cholesterol (HDL-C), and triglyceride levels. After centrifugation, one plasma specimen was kept and stored at $-80^{\circ} \mathrm{C}$ until measurement of LDL particle size as previously described. ${ }^{2}$ Briefly, LDL particle size was determined using a nondenaturing $2 \%$ to $16 \%$ polyacrylamide gradient gel electrophoresis. LDL particle size was extrapolated from the migration of standards of known diameters. In each gel, three standards containing four bands each were loaded. These plasma standards were previously calibrated with other standards of known diameters such as ferritin, thyroglobulin, and $380 \AA$ latex bands. LDL peak particle size corresponded to the estimated diameter of the major peak on the gel for each patient.

\section{Statistical Analysis}

Continuous data were expressed as mean \pm SEM and compared using unpaired $t$ test or a one-way analysis of variance to test the effect of group. Post hoc Tukey analyses were performed when the $P$ value of the analysis of variance was $<0.05$. Categorical data were expressed as a percentage and compared with the $\chi^{2}$ test. Correlations between variables were determined using Spearman coefficients. Multiple linear regression analysis was used to identify the independent correlates of BGN amount in AS valves. A $P$ value $<0.05$ was considered significant. Statistical analysis was performed with a commercially available software package JMP IN 7.1.

\section{Results}

\section{Microarray}

A tissue-based microarray experiment was first conducted to explore the gene expression pattern of LPL, LIPC, PLTP, and different proteoglycans on human aortic valves explanted from patients with or without AS. Five calcified AS valves and five control valves without any sign of calcification or dysfunction were selected for the microarray experiment. Cases and controls were white males, matched for age and body mass index absence of 
A

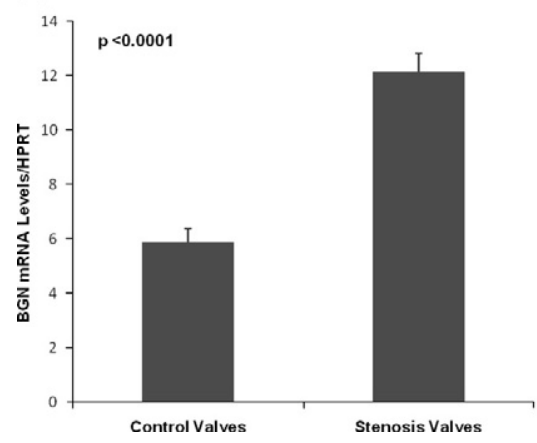

B

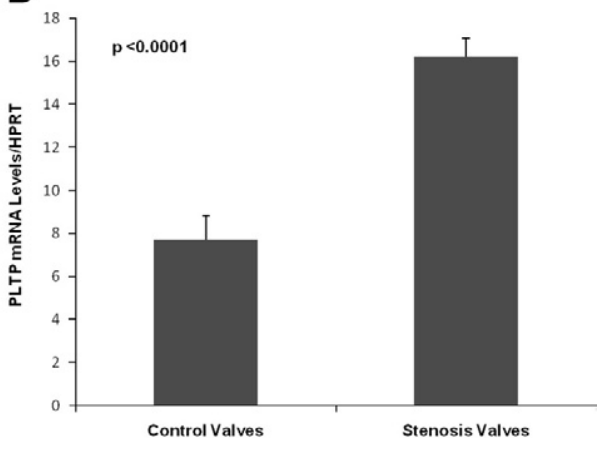

Figure 1. Biglycan (BGN) and phospholipid transfer protein (PLTP) mRNA transcript levels, measured with quantitative PCR, in aortic stenosis valves compared with control tissues (A and B). There was a significantly increased number of mRNA copies of BGN $(P<0.0001 ; \mathbf{A})$ and PLTP $(P<0.0001 ; \mathbf{B})$ in aortic stenosis valves compared with control noncalcified valves. renal failure and diabetes (Supplemental Table 1 at http://ajp.amjpathol.org). To reduce heterogeneity in the microarray experiment, bicuspid aortic valves were not used, and thus only AS valves with a tricuspid morphology were selected. Two control valves failed quality control and were discarded from subsequent analyses. The microarray results are presented in Supplemental Figure S1 at http://ajp.amjpathol.org. The heat map illustrates the normalized expression values for each sample. A total of 24 probe sets were available to study the expression of $B G N$, decorin (DCN), versican (CSPG2), perlecan (HSPG2), LPL, LIPC, and PLTP. The BGN and PLTP mRNA transcripts were significantly increased by 3.8-fold and 3.3-fold, respectively in AS compared with control valves. The other proteoglycans and lipid-modifying enzymes were not significantly different between AS and control valves.

Expression of BGN and PLTP has been conducted in a larger cohort of $87 \mathrm{AS}$ and 36 control valves to validate results of the microarray experiment. Using quantitative PCR, we found an increased number of copies of mRNA transcript for BGN $(P<0.0001)$ and PLTP $(P<0.0001)$ in $A S$ valves (Figure 1, $A$ and $B$ ).

\section{Expression of Biglycan, PLTP, and Their Relationships}

Using immunohistology, we documented a faint expression of BGN in control valves, whereas we found a strong immunoreactivity of this proteoglycan in AS valves (Figure 2, A and B). In AS valves, BGN was immunolocalized mainly in the fibrosa and the ventricularis. PLTP was immunolocalized to cells and was highly expressed in AS valves (Figure 2, C and D). Quantitative morphometric analyses of BGN and PLTP immunochemical expression confirmed the findings of the quantitative-PCR and revealed that $A S$ valves had a greater percentage area of immunomarking for BGN $(P<0.0001)$ and an increased cellular density of PLTP-positive cells $(P<0.0001)$ when compared with control valves (Figure 2, E and F). Of interest, we found that oxidized-LDL, PLTP, as well as Apo $\mathrm{Al}$ colocalized with $\mathrm{BGN}$ in the fibrosa layer of $\mathrm{AS}$ valves (Figure 3, A-D). Dual immunomarking confirmed that BGN and PLTP colocalized in AS valves (Figure 4A). In addition, we found that both BGN and PLTP colocalized with smooth muscle $\alpha$-actin and CD90, a fibroblast marker (Figure 4, B-E). Also, we documented that TLR 2 colocalized with both with BGN and PLTP (Figure 4, F and $G$ ), whereas TLR 4 colocalized only with BGN (Figure $4 \mathrm{H}$ ) and not PLTP (not shown). Levels of mRNA transcripts for BGN within AS valves had a significant and negative correlation with the LDL particle size $(r=-0.18$, $P=0.01$; Figure 5A). Of note, BGN was not associated with other lipid variables including plasma LDL, HDL, and triglyceride levels. In a multivariable model adjusted for age, gender, and triglyceride levels, LDL particle size $(P=0.008)$ was independently related to the amount of BGN transcripts in AS valve $\left(r^{2}\right.$ adjusted $=0.15, P=$
A

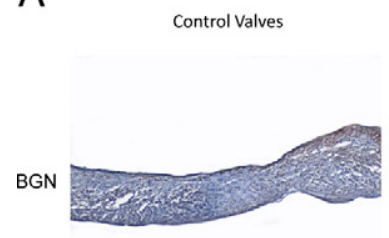

B

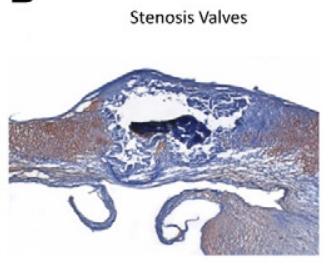

C

D
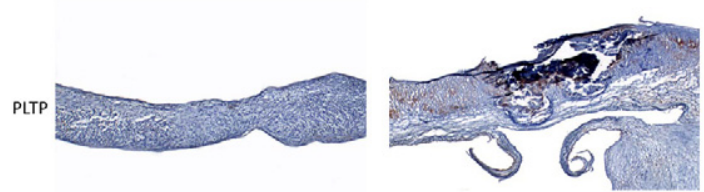

$\mathrm{E}_{40}$

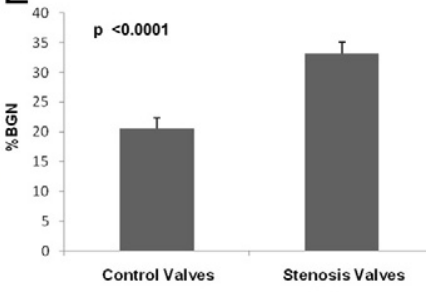

$F_{18}$

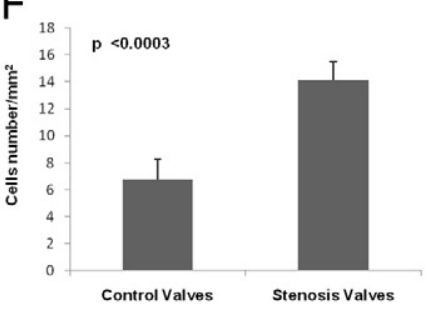

Figure 2. Immunolocalization of biglycan (BGN) and phospholipid transfer protein (PLTP) in control and aortic stenosis valves (A-D $\times 100$ ). In control valves BGN and PLTP were present in some areas but appear as a faint marking with respectively an extra-cellular and cellular localization (A and $\mathbf{C}$ ). In contrast, in aortic stenosis valves, BGN (B) and PLTP (D) were immunodetected and highly expressed, particularly in the fibrosa layer. Quantitative morphometric analyses revealed that AS valves had a greater percentage area of immunomarking for BGN and an increased cellular density of PLTP positive cells in AS valves when compared with control valves $(\mathbf{E}$ and $\mathbf{F})$ 
A

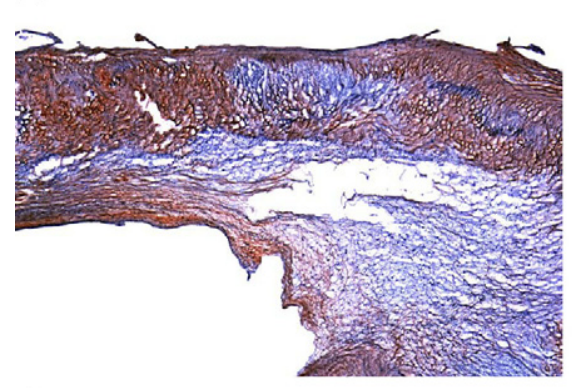

C

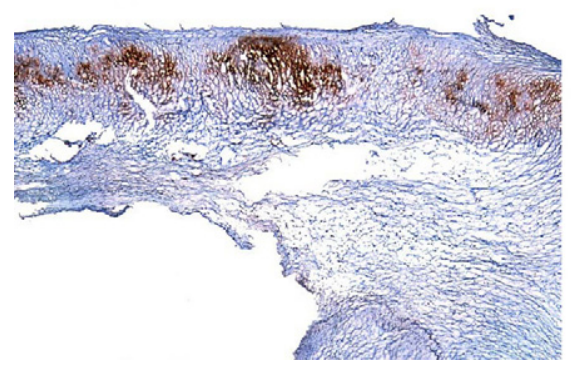

B

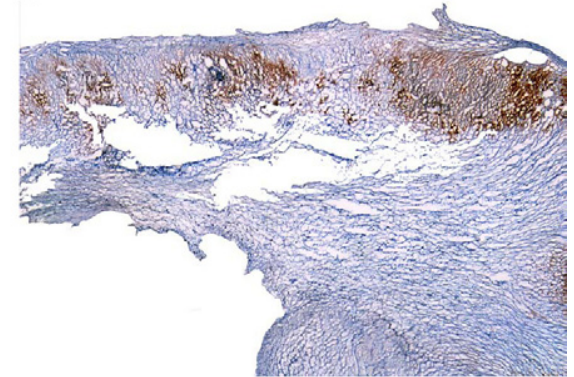

D

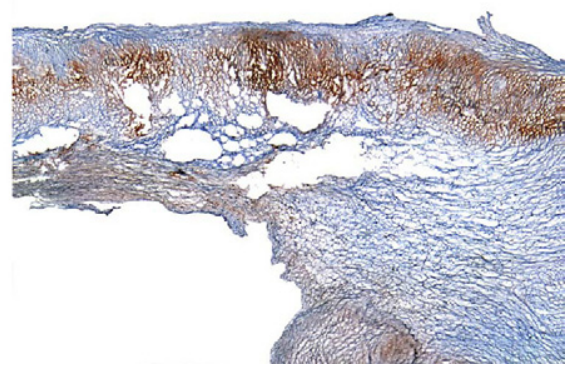

Figure 3. A-D: Immunohistochemistry studies showing the immunolocalization of biglycan (BGN), oxidized-LDL (ox-LDL), phospholipid transfer protein (PLTP), and apolipoprotein AI (Apo AI). BGN was immunolocalized in the fibrosa and ventricularis layers (A). Ox-LDL were detected and present in the fibrosa (B), where it colocalized with PLTP (C) and Apo AI (D $\times 200)$.
0.03). Of significance, the level of BGN in AS valves was also positively associated with the expression of PLTP $(r=0.48, P<0.0001)$ and NF- $\kappa \mathrm{B}(r=0.56, P<0.0001$; Figure 5, B and C). In turn, the expression of PLTP was significantly and positively correlated with NF- $\kappa$ B levels in AS valves $(r=0.43, P=0.001$; Figure $5 D)$.

\section{In Vitro Analyses with Valve Interstitial Cells}

Using isolated VICs we found that stimulation of cells during 48 hours with BGN $(100 \mathrm{ng} / \mathrm{ml})$ induced the production of PLTP at the protein level (Figure 6A). Of note, stimulation of VICs with PGN and LPS, respectively agonists of TLR 2 and TLR 4, also led to the synthesis of PLTP (Figure 6A). These results were then confirmed using quantitative PCR, which showed that increased number of PLTP copies was detected on stimulation with BGN, LPS, and PGN (Figure 6 B). Also, stimulation of VICs with BGN, LPS, and PGN led to an increased number of NF- $\kappa$ B transcripts (Figure $6 \mathrm{C}$ ). To further evaluate which TLR was involved in this response with $B G N$, we performed a series of experiments with the presence of anti-TLR 2 and 4 antibodies. With the presence of anti-TLR 2 antibody, the BGN-induced production of PLTP was abolished, whereas an irrelevant human antibody $\left(\operatorname{lgG}_{1}\right.$ FC) and an anti-TLR 4 antibody were without effect on this response (Figure 6D). In addition, anti-TLR 2 antibody also prevented BGN-induced NF- $\kappa \mathrm{B}$ mRNA synthesis (Figure $6 \mathrm{E})$. To corroborate these results we have also pretreated VICs with siRNA for TLR 2 and TLR 4. These experiments showed that BGN-mediated mRNA expression of PLTP and $\mathrm{NF}-\kappa \mathrm{B}$ were significantly reduced only with a pretreatment with siRNA for TLR 2 (Supplemental Figure S2, A and B at $h$ ttp://ajp.amjpathol.org). Also, NF- $\mathrm{B}$ activity after the stimulation of VICs by BGN, which was measured as the production of phospho NF- $\kappa \mathrm{B}$, was significantly reduced after a pretreatment of VICs with a siRNA for
TLR 2 ( $P=0.007$; Supplemental Figure S3, A and B at http://ajp.amjpathol.org).

\section{Discussion}

The findings of this study support that mechanisms allowing the retention of lipids are activated within AS valves and may contribute to disease activity. Furthermore, we have identified, for the first time, that BGN is a potent stimulator of PLTP production by VICs through TLR 2 and associated with NF- $\kappa$ B activation pathway. The observation that the size of LDL particle was independently and negatively related to the level of BGN in AS valves also suggests that intricate links exist between systemic lipoprotein metabolism and AS pathophysiology.

\section{The Role of Biglycan and PLTP in Aortic Stenosis}

Previous studies have underlined that BGN and PLTP are present and up-regulated in atherosclerotic plaques. ${ }^{9,10}$ BGN by its affinity to lipoproteins may contribute to atherosclerosis. ${ }^{11}$ According to the response-to-retention hypothesis, atherosclerosis is favored by mechanisms allowing lipids to remain within tissues. Among other mechanisms, it is suspected that retention of lipids will promote the production of oxidized lipid by-products in the atherosclerotic plaque. ${ }^{12}$ Hence, it is likely that similar mechanisms occur in AS valves and contribute to accumulation of ox-LDL. In the present study, the observation that BGN colocalized with ox-LDL is most consistent with this hypothesis. We have previously shown that accumulation of ox-LDL within AS valves is strongly associated with the activation of inflammatory pathways. ${ }^{2}$ Also of note, in the same study the only lipid variable associated 

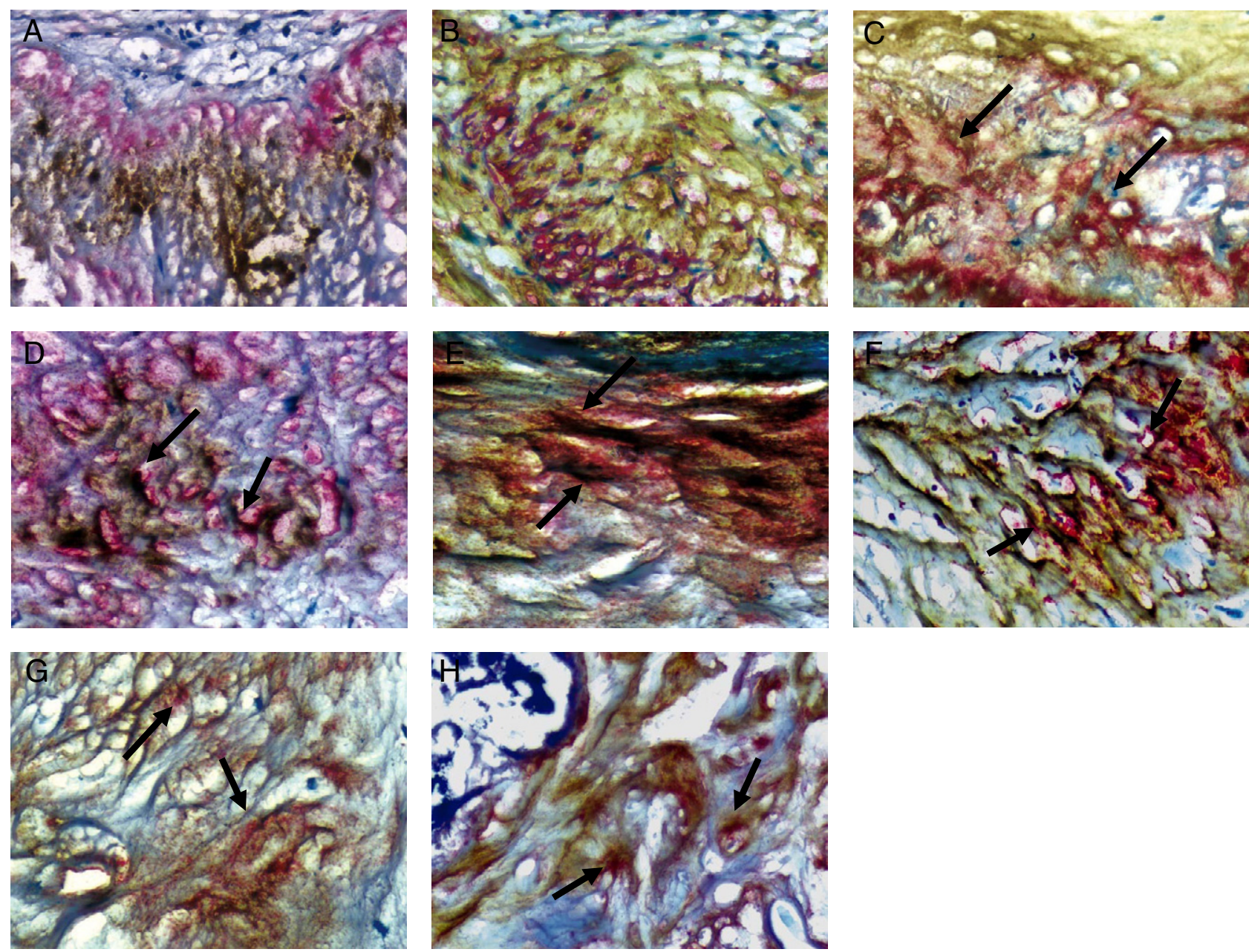

Figure 4. Double immununostainings confirmed that biglycan (BGN; red) colocalized with phospholipid transfer protein (PLTP; brown; A). In addition, we documented that BGN (brown) colocalized with smooth muscle $\alpha$-actin (red; B) along with CD90 (red; arrows; C). Also, PLTP (brown) was found to colocalize with smooth muscle $\alpha$-actin (red; arrows; D) and with CD90 (red; arrows; E). Furthermore, BGN (brown; F) and PLTP (brown; G) were documented to colocalize with TLR 2 (arrows; red). Of note, TLR 4 (red; arrows) was found to colocalize only with BGN (brown; H) and not PLTP (not shown).

with the accumulation of ox-LDL within AS valves was the level of plasma small dense LDL particles. In the present study we found a negative relationship between LDL particle size and BGN expression in AS valves. Although this remains to be further corroborated, these findings suggest that the increased proportion of small dense LDL particles in the plasma predisposed to the accumulation of oxidized lipids within the valvular tissue, which, in turn, led to the synthesis of BGN. Then, increased production of BGN would promote further lipid accumulation along with PLTP synthesis by VICs. In line with this, it has been documented that small dense LDLs have a greater rate of oxidation and that, in turn, oxidized products of ox-LDLs are potent inducers of BGN production in isolated cells. ${ }^{13,14}$

O'Brien et al have demonstrated that PLTP promotes modifications of Apo Al, which increase its binding affinities for proteoglycans. ${ }^{7}$ In this scheme of things, it is likely that trapping of modified HDLs within valvular tissue will reduce their ability to perform reverse cholesterol transport. Thus, synthesis of PLTP could promote lipid retention and thereby trigger valvular inflammation and mineralization. Among its many functions, PLTP also plays a determinant role in the production of circulating pre- $\beta$ HDL. ${ }^{15}$ Mice overexpressing PLTP have lower HDL levels and are prone to atherosclerosis. ${ }^{16,17}$ Also, mice with selective inhibition of PLTP production by macrophages exhibit fewer atherosclerotic lesions. ${ }^{18}$

\section{Toll-Like Receptors and Aortic Stenosis}

Recently, the presence of TLRs has been documented in VICs, the main cellular component of aortic valve. ${ }^{19}$ Moreover, studies indicate that TLRs may play a significant role in atherosclerosis. ${ }^{20}$ Different molecules have been shown to stimulate TLRs including ox-LDL. ${ }^{21}$ Furthermore, the recent discovery that endogenous matrix molecules, such as BGN, also stimulate murine TLR 2 and 4 in macrophages has cast a new light on the interaction between cellular matrix components and inflammatory cells. ${ }^{8}$ One of the novel findings of the present study is that BGN induces the production of PLTP by VICs through TLR 2. In this regard, the colocalization of TLR 2 with both PLTP and BGN in AS valves supports the in vitro studies with isolated VICs. In addition to its par- 
A

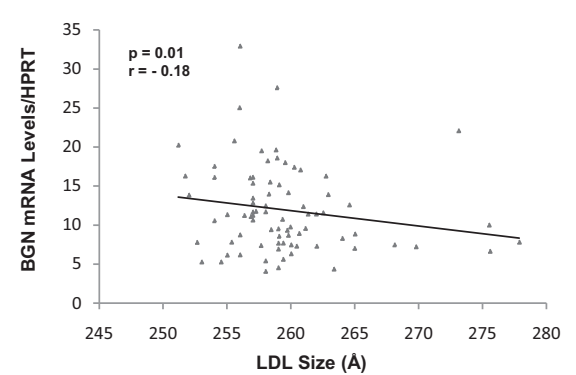

C

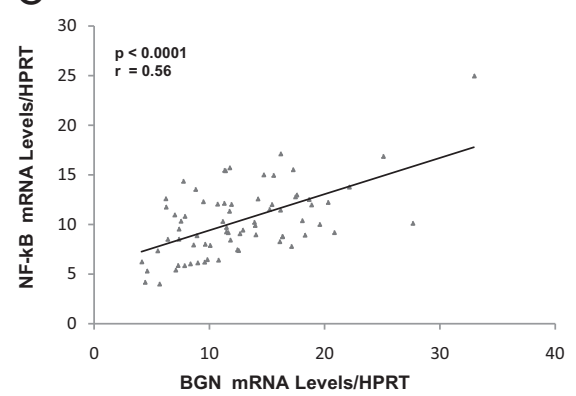

B

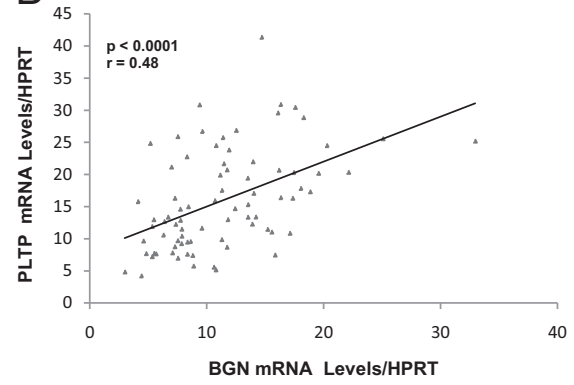

D

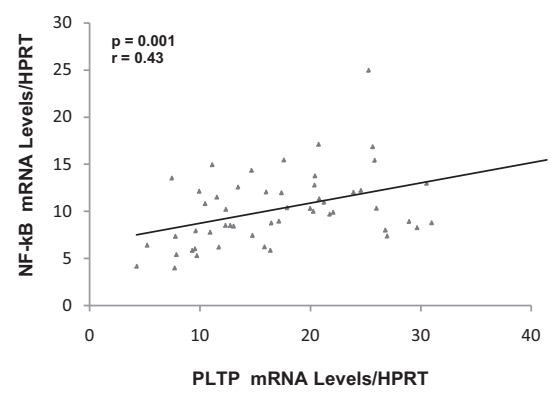

Figure 5. A-D: Correlations between biglycan (BGN), phospholipid transfer protein (PLTP), and nuclear factor kappa B (NF- $\kappa \mathrm{B})$ mRNA transcript levels along with plasma LDL particle size in aortic stenosis valves. BGN transcript level was negatively correlated with LDL particle size $(\mathrm{r}=-0.18, P=0.01 ; \mathbf{A})$. The mRNA transcript levels of BGN and PLTP were positively correlated $(r=0.48, P<0.0001 ; \mathbf{B})$. BGN and PLTP mRNA transcript levels were positively correlated with NF- $\kappa \mathrm{B}$ levels in aortic stenosis valves $(r=$ $0.56, P<0.0001 ; r=0.43, P=0.001 ; \mathbf{C}$ and $\mathbf{D})$. ticipation to tissue remodeling as well as to mineralization of tissues in AS valves, VICs can also be considered to play a major role in inflammation and the secretion of lipid-modifying proteins. In this regard, BGN-mediated PLTP synthesis by VICs is likely a mechanism involved in the metabolism of lipoproteins within AS valves. Thus, intricate mechanisms and interactions between extracellular matrix components, inflammation, and local metabolism of lipids is involved in the pathogenesis of AS.

\section{Clinical Implications}

So far, no medical treatment has been demonstrated to be efficient to alter the natural course of AS. ${ }^{22}$ In fact, the natural history of mild to moderate AS, while providing a time-window opportunity for treatment, is nonetheless prone to progression in a relatively short period of time, at least for a sizeable proportion of patients. Present findings and future studies may thus help to develop new

\section{A}

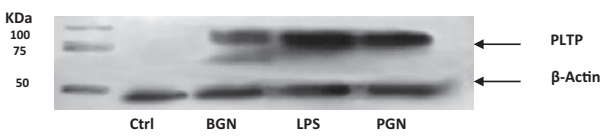

B

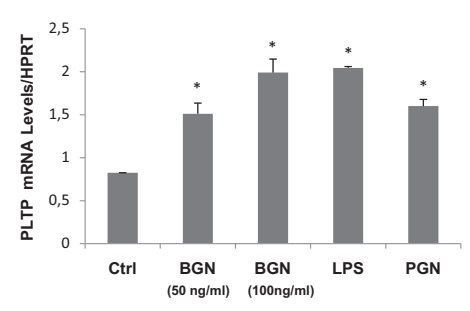

D

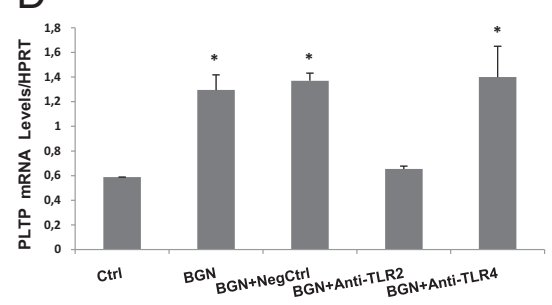

C

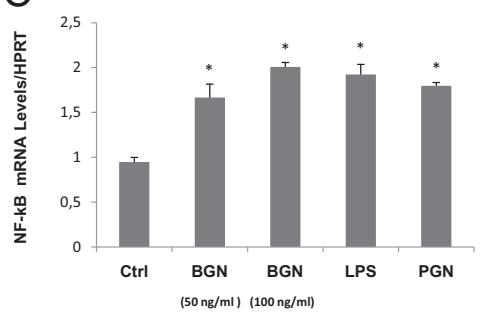

E

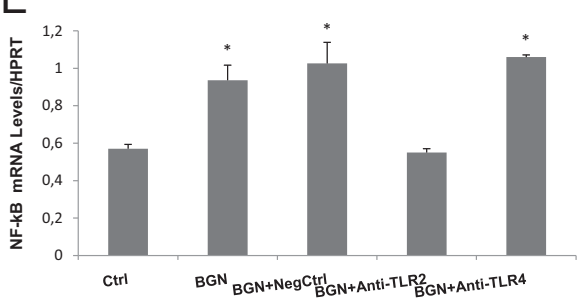

Figure 6. A-E: Expression of phospholipid transfer protein (PLTP) and nuclear factor kappa $\mathrm{B}(\mathrm{NF}-\kappa \mathrm{B})$ in isolated valve interstitial cells (VICs) on stimulation with biglycan (BGN), bacterial lipopolysaccharide (LPS), and peptidoglycan (PGN). Using immunoblotting we found that after 48 hours of stimulation with BGN (100 $\mathrm{ng} / \mathrm{ml})$, LPS $(200 \mathrm{ng} / \mathrm{ml})$, and PGN $(10 \mu \mathrm{g} / \mathrm{ml})$, PLTP was produced by VICs (A). Also, mRNA transcript levels of PLTP and NF- $\kappa$ B were significantly increased on stimulation for 48 hours with BGN, LPS, and PGN (B and $\mathbf{C})$. Addition of anti-Toll receptor 2 and anti-Toll receptor 4 antibodies in the medium of stimulated cells with BGN revealed that the BGN-induced production of PLTP and NF- $\kappa$ B were abrogated only by anti-Toll receptor 2 antibody $(\mathbf{D}$ and $\mathbf{E}) .{ }^{*} P<$ 0.01 compared with control (cntrl); BGN + NegCtrl: biglycan and an irrelevant human control antibody. 
treatments to prevent the progression of this disorder. The trafficking of lipids along with their modifications once within the AS valve may represent a promising target of therapy. To this effect, we have identified an independent association between the size of LDL particles in the plasma and the amount of BGN within AS valves. It is thus possible that increasing the size of $L D L$ particles with the use of lifestyle modification program and/or pharmacological interventions would translate into lower synthesis of BGN and PLTP. This would ultimately result in a reduction of lipid accumulation within the valvular tissue, a process thought to play an important role in inflammation and mineralization of AS valves. In addition, although still experimental, newer therapy aimed at reducing interactions between lipids and proteoglycans might be also envisioned. ${ }^{23}$ Moreover, although still in its infancy, the development of TLRs antagonists may provide novel alternative therapy. ${ }^{24}$ Further studies in animal models of AS are, however, necessary to validate these potential therapeutic avenues.

\section{Limitations}

In the present study, tissue expression of BGN and PLTP were conducted in patients having a severe AS. Thus, conclusions cannot necessarily be extrapolated to the disease pattern in mild to moderate AS. These findings nonetheless indicate that the mechanisms identified in this study are involved in the end stage of AS and are, therefore, relevant to the understanding of the pathobiology of AS.

\section{Conclusions}

In this study we have found increased expression of BGN and PLTP within AS valves. Furthermore, we have documented that BGN stimulates the synthesis of PLTP by VICs through TLR 2. Hence, mechanisms participating in lipid modification and retention are present within AS valves and may represent an important pathophysiological process. Further studies may help to delineate the relationships between valve matrix biology, inflammation, and lipid retention. This may, in turn, lead to the development of novel strategies to treat calcific AS.

\section{References}

1. Rajamannan NM: Calcific aortic stenosis: lessons learned from experimental and clinical studies. Arterioscler Thromb Vasc Biol 2009, 29:162-168

2. Mohty D, Pibarot P, Despres JP, Cote C, Arsenault B, Cartier A, Cosnay P, Couture C, Mathieu P: Association between plasma LDL particle size, valvular accumulation of oxidized LDL, and inflammation in patients with aortic stenosis. Arterioscler Thromb Vasc Biol 2008, 28:187-193

3. Meng X, Ao L, Song Y, Babu A, Yang X, Wang M, Weyant MJ, Dinarello CA, Cleveland JC Jr, Fullerton DA: Expression of functional Toll-like receptors 2 and 4 in human aortic valve interstitial cells: potential roles in aortic valve inflammation and stenosis. Am J Physiol Cell Physiol 2008, 294:C29-C35

4. Yang X, Fullerton DA, Su X, Ao L, Cleveland JC Jr, Meng X: Proosteogenic phenotype of human aortic valve interstitial cells is associated with higher levels of Toll-like receptors 2 and 4 and enhanced expression of bone morphogenetic protein 2. J Am Coll Cardiol 2009, 53:491-500

5. Gustafsson M, Boren J: Mechanism of lipoprotein retention by the extracellular matrix. Curr Opin Lipidol 2004, 15:505-514

6. Tabas I, Williams KJ, Boren J: Subendothelial lipoprotein retention as the initiating process in atherosclerosis: update and therapeutic implications. Circulation 2007, 116:1832-1844

7. O'Brien KD, Vuletic S, McDonald TO, Wolfbauer G, Lewis K, Tu AY, Marcovina S, Wight TN, Chait A, Albers JJ: Cell-associated and extracellular phospholipid transfer protein in human coronary atherosclerosis. Circulation 2003, 108:270-274

8. Schaefer L, Babelova A, Kiss E, Hausser HJ, Baliova M, Krzyzankova M, Marsche G, Young MF, Mihalik D, Gotte M, Malle E, Schaefer RM, Grone $\mathrm{HJ}$ : The matrix component biglycan is proinflammatory and signals through Toll-like receptors 4 and 2 in macrophages. J Clin Invest 2005, 115:2223-2233

9. Kunjathoor VV, Chiu DS, O'Brien KD, LeBoeuf RC: Accumulation of biglycan and perlecan, but not versican, in lesions of murine models of atherosclerosis. Arterioscler Thromb Vasc Biol 2002, 22:462-468

10. Desrumaux CM, Mak PA, Boisvert WA, Masson D, Stupack D, Jauhiainen M, Ehnholm C, Curtiss LK: Phospholipid transfer protein is present in human atherosclerotic lesions and is expressed by macrophages and foam cells. J Lipid Res 2003, 44:1453-1461

11. O'Brien KD, Lewis K, Fischer JW, Johnson P, Hwang JY, Knopp EA, Kinsella MG, Barrett PH, Chait A, Wight TN: Smooth muscle cell biglycan overexpression results in increased lipoprotein retention on extracellular matrix: implications for the retention of lipoproteins in atherosclerosis. Atherosclerosis 2004, 177:29-35

12. Williams KJ, Tabas I: The response-to-retention hypothesis of early atherogenesis. Arterioscler Thromb Vasc Biol 1995, 15:551-561

13. Tribble DL, Holl LG, Wood PD, Krauss RM: Variations in oxidative susceptibility among six low density lipoprotein subfractions of differing density and particle size. Atherosclerosis 1992, 93:189-199

14. Chang MY, Tsoi C, Wight TN, Chait A: Lysophosphatidylcholine regulates synthesis of biglycan and the proteoglycan form of macrophage colony stimulating factor. Arterioscler Thromb Vasc Biol 2003, 23:809-815

15. Settasatian N, Duong M, Curtiss LK, Ehnholm C, Jauhiainen M, Huuskonen J, Rye KA: The mechanism of the remodeling of high density lipoproteins by phospholipid transfer protein. J Biol Chem 2001, 276:26898-26905

16. Yang XP, Yan D, Qiao C, Liu RJ, Chen JG, Li J, Schneider M, Lagrost $L$, Xiao X, Jiang XC: Increased atherosclerotic lesions in apoE mice with plasma phospholipid transfer protein overexpression. Arterioscler Thromb Vasc Biol 2003, 23:1601-1607

17. Lie J, de CR, van GT, van HR, Scheek L, Sadeghi-Niaraki F, van TA: Elevation of plasma phospholipid transfer protein increases the risk of atherosclerosis despite lower apolipoprotein B-containing lipoproteins. J Lipid Res 2004, 45:805-811

18. Vikstedt R, Ye D, Metso J, Hildebrand RB, Van Berkel TJ, Ehnholm C Jauhiainen M, Van EM: Macrophage phospholipid transfer protein contributes significantly to total plasma phospholipid transfer activity and its deficiency leads to diminished atherosclerotic lesion development. Arterioscler Thromb Vasc Biol 2007, 27:578-586

19. Babu AN, Meng X, Zou N, Yang X, Wang M, Song Y, Cleveland JC, Weyant M, Banerjee A, Fullerton DA: Lipopolysaccharide stimulation of human aortic valve interstitial cells activates inflammation and osteogenesis. Ann Thorac Surg 2008, 86:71-76

20. Xu XH, Shah PK, Faure E, Equils O, Thomas L, Fishbein MC, Luthringer D, Xu XP, Rajavashisth TB, Yano J, Kaul S, Arditi M: Toll-like receptor-4 is expressed by macrophages in murine and human lipid-rich atherosclerotic plaques and upregulated by oxidized LDL. Circulation 2001, 104:3103-3108

21. Miller YI, Viriyakosol S, Worrall DS, Boullier A, Butler S, Witztum JL: Toll-like receptor 4-dependent and -independent cytokine secretion induced by minimally oxidized low-density lipoprotein in macrophages. Arterioscler Thromb Vasc Biol 2005, 25:1213-1219

22. Otto CM: Calcific aortic stenosis-time to look more closely at the valve. N Engl J Med 2008, 359:1395-1398

23. Khalil MF, Wagner WD, Goldberg IJ: Molecular interactions leading to lipoprotein retention and the initiation of atherosclerosis. Arterioscler Thromb Vasc Biol 2004, 24:2211-2218

24. Erickson B, Sperber K, Frishman WH: Toll-like receptors: new therapeutic targets for the treatment of atherosclerosis, acute coronary syndromes, and myocardial failure. Cardiol Rev 2008, 16:273-279 